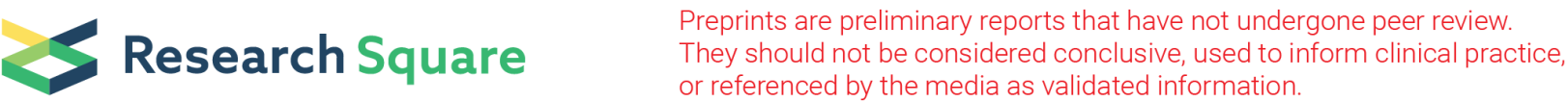

\section{Effect on 30-day Mortality and Duration of Hospitalization of Empirical Antibiotic Therapy in CRGNB-Infected Pneumonia}

rongrong li

Hefei Second People's Hospital

Hao Tang

Fourth Affiliated Hospital of Anhui Medical University

Huaming $X u$

Fourth Affiliated Hospital of Anhui Medical University

Kunwei Cui

First Affiliated Hospital of Anhui Medical University

Shujin Li

Hefei Second People's Hospital

Jilu Shen (D2565989451@qq.com )

First Affiliated Hospital of Anhui Medical University laboratory

\section{Research}

Keywords: appropriate empirical antibiotics treatment, bacterial pneumonia, carbapenemase-producing, gram-negative bacteria, mortality

Posted Date: January 15th, 2021

DOl: https://doi.org/10.21203/rs.3.rs-146124/v1

License: (9) This work is licensed under a Creative Commons Attribution 4.0 International License.

Read Full License

Version of Record: A version of this preprint was published on March 7th, 2021. See the published version at https://doi.org/10.1186/s12941-021-00421-2. 


\section{Abstract}

\section{Purpose}

The objective of this study was to investigate whether unreasonable empirical antibiotic treatment(UEAT) had an impact on 30-day mortality and duration of hospitalization in bacterial pneumonia caused by carbapenem-resistant gram-negative bacteria (CRGNB).

\section{Methods}

This was a retrospective study involving CRGNB-infected pneumonia. All patients received empirical and targeted antibiotic treatment. The exposure variable was treated with empirical antibiotic treatment(EAT) within 48 hours of incubation and the outcome was 30-day mortality and duration of hospitalization. Moreover, we also considered other variables that might be relevant and conducted multivariable regression analysis of 30-day mortality and duration of hospitalization.

\section{Results}

The experiment collected 310 cases, the most common bacterium is Acinetobacter baumannii $(211 / 310$ [68\%]) and the others were Klebsiella pneumoniae(46/310 [15\%]) Pseudomonas aeruginosa and others (53/310 [17\%]). 76/310 (24.5\%) patients received appropriate empirical antibiotic treatment(REAT). In the analysis of risk factors, dementia, unconsciousness were risk factors of 30-day mortality and pulmonary disease, hemodynamic support at culture taken day and recent surgery were risk factors for longer hospital stay. 30-day mortality was 9 of 76(11.84\%) with REAT vs 36 of 234 (15.38\%) with UEAT (P $=0.447)$, UEAT was not associated with 30-day mortality. On the contrary, there was difference between REAT and UEAT $(P=0.023)$ in the analysis of EAT on hospitalization time.

\section{Conclusions}

UEAT was not associated with 30-day mortality while was related to duration of hospitalization in CRGNB-infected pneumonia, in which Acinetobacter baumanniii accouned for the majority.

\section{Background}

EAT was carried out before the identification of bacteria and the determination of drug susceptibility. EAT for infections might be reasonable or unreasonable, that was, in vitro sensitivity with subsequently isolated pathogens, whether sensibility matched or not. For patients with severe infection, most clinicians tended to start EAT in the early stage of infection and the treatment scheme was often combined therapy ${ }^{[1]}$. At present, EAT has existed in the treatment of various infections, including bloodstream infections, urinary tract infections, pulmonary infections and so on ${ }^{[2-9]}$, but the efficacy of EAT is not entirely certain. and the excessive or unreasonable use of antibiotics were related to the increase of bacterial resistance, side effects and treatment costs. These problem were related to the treatment of infected patients by clinicians ${ }^{[10]}$. 
Patients admitted to ICU were prone to hospital-acquired pneumonia because of many underlying diseases, poor surrounding environment and low autoimmunity ${ }^{[11]}$. For some patients who needed catheter insertion, such as nasal catheter, mask, tracheal intubation and so on, bacteria easily entered the body through the catheter cavity and lead to catheter-related infection finally. The untime treatment of such infection often resulted in death ${ }^{[12]}$. Based on this background, we studied the effects of EAT on $30-$ day mortality and hospital length of stay.

\section{Methods}

\section{Study Design}

This was a retrospective study, cases were collected from January 2014 to March 2019 at Department of Laboratory Medicine,The First Affiliated Hospital of Anhui Medical University, a 2825-bed, tertiary teaching hospital in China. The first positive culture of each patient was taken as one sample in this study. The study was approved by the ethics committee of participating hospital.

\section{Participants}

Our experiment collected adult inpatients $\geq 18$ years with hospital-acquired bacterial pneumonia or ventilator-associated bacterial pneumonia caused by CRGNB: Acinetobacter baumannii, Pseudomonas aeruginosa, or any Enterobacteriaceae. All inpatients received EAT and targeted antibiotic therapy.

\section{Definitions}

REAT was defined as antibiotic therapy used within 48 hours of bacterial culture and bacteria were sensitive to the antibiotics used in vitro drug sensitivity tests. 30-day mortality was defined as all-cause mortality at 30 days after the first positive culture isolate was taken from the patient. Hospitalization time was the number of days from the onset of infection to the discharge or death of patients with hospitalacquired infections. There were two clinical criteria for the diagnosis of bacterial pneumonia. First, there were obvious symptoms of cough and sputum, even fever, chest pain and so on. Second, chest X-ray or chest CT showed obvious patchy shadows in the lungs.

\section{Variables}

The exposure variable was EAT and the outcome was all-cause mortality at 30 days and hospital stay. Data were ascertained from patients written and electronic records and microbiology laboratory records. Besides, we considered other variables that might affect 30-day mortality and hospital stay, including patient demographics, basic disease, laboratory tests, unconsciousness, hemodynamic support, catheters, bacterial types, recent surgery and so on.

Microbiology Methods

Isolates were identified by MALDI-TOF MS systems( Bio Mérieux囚Marcy I 'Etoile『France). Antibiotic susceptibilities testing was performed by Clinical and Laboratory Standards Institute(CLSI) guidelines 2018. The broth dilution method was used to analyze the sensitivity of Acinetobacter baumannii and 
Enterobacteriaceae to tegacyclin according to the interpretation standard established by FDA, When the minimum inhibitory concentration were less than or equal to $2 \mu \mathrm{g} / \mathrm{ml}, 4 \mu \mathrm{g} / \mathrm{ml}$ and more than $8 \mu \mathrm{g} / \mathrm{ml}$, it was sensitive, intermediate and resistant separately.

\section{Statistical Analysis}

We categorized the collected cases and compared data on survivors and deaths, as well as hospitalization time less than or equal to 30 days and more than 30 days. And proportions were compared using a Fisher exact test or chi-square test, continuous variables using t test or Mann-Whitney $U$ test according to their distribution, the results were expressed as mean \pm standard deviation and median (interquartile range) respectively. Risk factors for 30-day mortality or duration of hospitalization found significant on univariate analysis were entered into a multivariable logistic regression. Predictive performance of the model was assessed using Odds ratios (ORs) with 95\% confidence intervals (Cls). Analyses were conducted using SPSS version 23.0 software.

\section{Results}

A total of 310 eligible cases were collected, of which 76 (24.5\%) were given REAT and the remaining 234 (75.5\%) were UEAT. There were 105 (33.9\%) female cases. The mean average age was $59.54 \pm 16.59$ years. Among them, Acinetobacter baumannii, Klebsiella pneumoniae, Pseudomonas aeruginosa and others were isolated from 211, 46 and 53 patients respectively, The division distribution and drug resistance of all cases were shown in Table 1 and Table 2, the cases collected were mainly concentrated in emergency department(183/310). Drug sensitivity showed that Acinetobacter baumannii and Klebsiella pneumoniae had high resistance to most antibiotics, and Pseudomonas aeruginosa present different degrees of resistance to each antibiotic. Similarly, all three bacteria showed low resistance to tegacycline, minocycline and polymyxin( $<8 \%)$.

Table 1. The division distribution of all cases 


\begin{tabular}{|c|c|}
\hline Departments & Number \\
\hline Nephrology & 1 \\
\hline Gastrointestinal Surgery & 1 \\
\hline Cardiac Surgery & 11 \\
\hline Cardiac Surgery ICU & 16 \\
\hline Thoracic Surgery & 14 \\
\hline Hematology Department & 15 \\
\hline Ophthalmology Department & 1 \\
\hline Plastic surgery & 3 \\
\hline Oncology Department of Integrated Traditional Chinese and Western Medicine & 1 \\
\hline Traditional Chinese Medicine Department & 1 \\
\hline Department of Critical Care Medicine & 67 \\
\hline Hepatobiliary Surgery & 3 \\
\hline Infectious Disease Department & 9 \\
\hline Cadre ICU & 27 \\
\hline orthopaedics & 2 \\
\hline Respiratory Department & 28 \\
\hline Emergency ICU & 61 \\
\hline Emergency Department of Internal Medicine. & 11 \\
\hline emergency surgery & 1 \\
\hline Urology Surgery & 8 \\
\hline The skin Department & 1 \\
\hline Burn Department & 3 \\
\hline Internal Medicine-Neurology & 16 \\
\hline neurosurgery & 9 \\
\hline
\end{tabular}

Abbreviations: ICU, intensive care unit.

Table 2. The drug resistance of all bacteria 


\begin{tabular}{|c|c|c|c|}
\hline \multirow[t]{2}{*}{ Antibiotics } & \multicolumn{3}{|c|}{ Drug resistance rate } \\
\hline & $\begin{array}{l}\text { Acinetobacter } \\
\text { baumannii }\end{array}$ & $\begin{array}{l}\text { Klebsiella } \\
\text { pneumoniae }\end{array}$ & $\begin{array}{l}\text { Pseudomonas } \\
\text { aeruginosa }\end{array}$ \\
\hline Ertapenem & / & $93.75 \%$ & / \\
\hline Ampicillin & / & $100.00 \%$ & / \\
\hline Ampicillin/sulbactam & $94.50 \%$ & $100.00 \%$ & / \\
\hline Nitrofurantoin & / & $96.55 \%$ & / \\
\hline $\begin{array}{l}\text { Compound } \\
\text { sulfamethoxazole }\end{array}$ & $72.86 \%$ & $47.50 \%$ & / \\
\hline Ciprofloxacin & $91.90 \%$ & $73.91 \%$ & $50.00 \%$ \\
\hline Piperacillin/tazobactam & $88.68 \%$ & $67.39 \%$ & $39.13 \%$ \\
\hline Gentamicin & $75.24 \%$ & $67.39 \%$ & $40.82 \%$ \\
\hline Cefepime & $94.29 \%$ & $84.78 \%$ & $42.31 \%$ \\
\hline Ceftriaxone & $95.24 \%$ & $97.50 \%$ & / \\
\hline Ceftazidime & $94.76 \%$ & $89.13 \%$ & $55.10 \%$ \\
\hline Cefotetan & l & $100.00 \%$ & / \\
\hline Cefoxitin & l & $100.00 \%$ & I \\
\hline Tobramycin & $70.81 \%$ & $65.22 \%$ & $43.14 \%$ \\
\hline Imipenem & $98.57 \%$ & $89.13 \%$ & $96.08 \%$ \\
\hline Cefuroxime & I & $100.00 \%$ & I \\
\hline Tigecycline & $2.99 \%$ & $3.85 \%$ & I \\
\hline Cefoperazone/sulbactam & I & $73.33 \%$ & I \\
\hline Levofloxacin & $48.80 \%$ & $71.74 \%$ & $38.46 \%$ \\
\hline Cefotaxime & $95.83 \%$ & $96.15 \%$ & I \\
\hline Minocycline & $7.88 \%$ & $0.00 \%$ & I \\
\hline Meropenem & $96.91 \%$ & $97.30 \%$ & $83.33 \%$ \\
\hline Cefmetazole & I & $75.00 \%$ & I \\
\hline Piperacillin & $97.67 \%$ & $76.92 \%$ & $43.48 \%$ \\
\hline Cefazolin & l & $100.00 \%$ & / \\
\hline Amikacin & $57.67 \%$ & $63.04 \%$ & $22.00 \%$ \\
\hline
\end{tabular}




\begin{tabular}{|llll|} 
Polymyxin & $0.60 \%$ & $0.00 \%$ & $3.33 \%$ \\
\hline Aztreonam & $/$ & $97.67 \%$ & $75.00 \%$ \\
\hline
\end{tabular}

\section{Factors Associated With Duration of Hospitalization}

In this study, we listed risk factors that may be associated with 30-day mortality and duration of hospitalization, which were shown in Table 3 and Table 6. As shown in Table 3. For the analysis of hospitalization time, we categorized hospitalization time according to whether it was longer than 30 days and compared the two categories. Firstly, in univariate analysis, risk factors for hospitalization longer than 30 days were age $\llbracket$ dementia[pulmonary disease $\square$ hemodynamic support at culture taken dayロarterial line $\llbracket$ central line $\llbracket$ acquisition in ICU and recent surgery. Secondly, the result of multivariate regression analysis(Table 4) showed that pulmonary diseaselhemodynamic support at culture taken day and recent surgery were risk factors for longer hospital stay. Finally, we divided EAT into REAT and UEAT to analyze the effect of EAT on hospitalization time(Table 5). The result showed that there was significant difference in length of stay between REAT and UEAT $(P=0.023)$.

Table 3. Factors Associated With Duration Of Hospitalization 


\begin{tabular}{|c|c|c|c|}
\hline & $\leq 30$ days & $>30$ days & $\mathrm{P}$ \\
\hline Factors & $\bigotimes n=146 \rrbracket$ & $\triangle n=164 \rrbracket$ & \\
\hline Age & $62.09 \pm 17.88$ & $57.28 \pm 15.05$ & 0.011 \\
\hline Gender & & & 0.556 \\
\hline Male & $99(67.81 \%)$ & $106(64.63 \%)$ & \\
\hline female & $47(32.19 \%)$ & $58(35.37 \%)$ & \\
\hline $\operatorname{BMI}\left(\mathrm{Kg} / \mathrm{m}^{\wedge} 2\right)$ & $21.16 \pm 4.31(42)$ & $22.61 \pm 3.72(83)$ & 0.054 \\
\hline Congestive heart failure & $31(21.23 \%)$ & $37(22.56 \%)$ & 0.778 \\
\hline Dementia & $6(4.11 \%)$ & $1(0.61 \%)$ & 0.038 \\
\hline Pulmonary disease & $21(14.38 \%)$ & $3(1.83 \%)$ & 0.000 \\
\hline Active malignancy & & & 0.499 \\
\hline None & 127(86.97\%) & $135(82.32 \%)$ & \\
\hline Solid & $15(10.27 \%)$ & $24(14.63 \%)$ & \\
\hline Hematological & $4(2.74 \%)$ & $5(3.05 \%)$ & \\
\hline Liver disease & & & 0.907 \\
\hline None & 137(93.84\%) & $152(92.68 \%)$ & \\
\hline Mild & $8(5.48 \%)$ & $11(6.71 \%)$ & \\
\hline Severe & $1(0.68 \%)$ & $1(0.61 \%)$ & \\
\hline Diabetes mellitus with end-organ damage & $1(0.68 \%)$ & $2(1.22 \%)$ & 0.631 \\
\hline Renal disease & $29(19.86 \%)$ & $38(23.17 \%)$ & 0.480 \\
\hline Total Charlson score & $2 \bigotimes 1-3 \rrbracket$ & $2 \bigotimes 1-3 \rrbracket$ & 0.033 \\
\hline Creatinine $(\mathrm{mg} / \mathrm{dL})^{\mathrm{a}}$ & $66.5 \rrbracket 48-106.55 \rrbracket$ & $65.5 \rrbracket 47.1-139.4 \rrbracket$ & 0.695 \\
\hline Albumin $(\mathrm{g} / \mathrm{dL})^{a}$ & $33.54 \pm 6.09$ & $34.01 \pm 6.15$ & 0.502 \\
\hline WBC $(\times 109 / L)^{a}$ & $11.26 \pm 4.99$ & $11.06 \pm 5.2$ & 0.584 \\
\hline Systolic blood pressure $(\mathrm{mm} \mathrm{Hg})^{a}$ & $126.01 \pm 18.62$ & $122.46 \pm 17.4$ & 0.084 \\
\hline Hemodynamic support $^{a}$ & $57(39.04 \%)$ & 115(70.12\%) & 0.000 \\
\hline Invasive ventilator support ${ }^{\mathrm{a}}$ & $83(56.85 \%)$ & $105(64.02 \%)$ & 0.197 \\
\hline Normal consciousness & $68(46.58 \%)$ & $82(50 \%)$ & 0.547 \\
\hline
\end{tabular}




\begin{tabular}{|c|c|c|c|}
\hline Arterial line & $25(17.12 \%)$ & $47(28.66 \%)$ & 0.016 \\
\hline Urine catheter & $106(72.60 \%)$ & $132(80.49 \%)$ & 0.101 \\
\hline Central line & $57(39.04 \%)$ & $88(53.66 \%)$ & 0.010 \\
\hline Nasogastric tube & $113(77.40 \%)$ & $131(79.88 \%)$ & 0.594 \\
\hline Acquisition in ICU & $69(47.26 \%)$ & $96(58.54 \%)$ & 0.047 \\
\hline Recent surgery & $43(29.45 \%)$ & $89(54.27 \%)$ & 0.000 \\
\hline Type of bacteria & & & 0.623 \\
\hline Acinetobacter & $101(69.18 \%)$ & $128(78.05 \%)$ & \\
\hline Enterobacteriaceae (Klebsiella) & $16(10.96 \%)$ & $19(11.58 \%)$ & \\
\hline Pseudomonas/other & $29(19.86 \%)$ & $17(10.37 \%)$ & \\
\hline EAT & & & 0.266 \\
\hline REAT & $40(27.40 \%)$ & $36(21.95 \%)$ & \\
\hline UEAT & $106(72.60 \%)$ & $128(78.05 \%)$ & \\
\hline Covering empirical therapy by time & & & 0.681 \\
\hline Same day as culture & $32(21.92 \%)$ & $26(15.85 \%)$ & \\
\hline Day +1 & $5(3.42 \%)$ & $5(3.05 \%)$ & \\
\hline Day +2 & $3(2.05 \%)$ & $5(3.05 \%)$ & \\
\hline
\end{tabular}

Data are presented as No. (\%) unless otherwise indicated.

aat culture taken day,

Abbreviations: BMI, body mass index; WBC, white blood cell; EAT: empirical antibiotic treatment; REAT: reasonable empirical antibiotic treatment; UEAT: unreasonable empirical antibiotic treatment; ICU, intensive care unit.

Table 4. Risk Factors For Duration Of Hospitalization, Multivariate Analysisa 


\begin{tabular}{|lllll|}
\hline & \multicolumn{5}{c|}{$95 \% \mathrm{Cl}$} \\
\hline Age & OR & Lower & Upper & P \\
\hline Dementia & 0.995 & 0.98 & 1.01 & 0.511 \\
\hline Pulmonary disease & 7.686 & 0.816 & 72.437 & 0.075 \\
\hline Hemodynamic support at culture taken day & 0.389 & 0.219 & 0.691 & 0.001 \\
\hline Arterial line & 5.049 & 1.395 & 18.274 & 0.014 \\
\hline Central line & 0.784 & 0.388 & 1.585 & 0.498 \\
\hline Acquisition in ICU & 0.844 & 0.48 & 1.484 & 0.555 \\
\hline Recent surgery & 0.948 & 0.537 & 1.674 & 0.855 \\
\hline
\end{tabular}

Table 5. Comparison of hospitalization time between REAT and UEAT

\begin{tabular}{|c|c|c|c|}
\hline Factor & REAT $₫ n=76 \rrbracket$ & UEAT $\otimes n=234 \rrbracket$ & $P$ \\
\hline Duration of hospitalization & $31.68 \pm 11.56$ & $34 \llbracket 21-49.25 \rrbracket$ & 0.023 \\
\hline
\end{tabular}

Table 6. Risk Factors for 30-day Mortality 


\begin{tabular}{|c|c|c|c|}
\hline & Alive & Dead & \\
\hline Factors & $(n=265)$ & $(n=45)$ & $P$ \\
\hline Age, mean \pm SD & $59.19 \pm 16.24$ & $61.62 \pm 18.57$ & 0.365 \\
\hline Gender & & & 0.075 \\
\hline Male & $170 \rrbracket 64.2 \rrbracket$ & $35(77.8)$ & \\
\hline female & $95 \rrbracket 35.8 \rrbracket$ & $10(22.2)$ & \\
\hline $\mathrm{BMl}, \mathrm{kg} / \mathrm{m} 2$ & $22.17 \pm 4.98(16)$ & $22.12 \pm 3.85(109)$ & 0.964 \\
\hline Congestive heart failure & $53 \llbracket 20.0 \rrbracket$ & 15(33.3) & 0.046 \\
\hline Dementia & $3 \otimes 1.1 \rrbracket$ & $4(8.9)$ & 0.001 \\
\hline Pulmonary disease & $20 \otimes 7.5 \rrbracket$ & $4(8.9)$ & 0.756 \\
\hline Active malignancy & & & 0.072 \\
\hline None & $220 \rrbracket 83.0 \rrbracket$ & $42(93.3)$ & \\
\hline Solid & $36 \bigotimes 13.6 \rrbracket$ & $3(6.7)$ & \\
\hline Hematological & $9 \rrbracket 3.4 \rrbracket$ & 0 & \\
\hline Liver disease & & & 0.965 \\
\hline None & 247ه93.2】 & $42(93.3)$ & \\
\hline Mild & $16 \rrbracket 6.0 \rrbracket$ & $3(6.7)$ & \\
\hline Severe & $2 \bowtie 0.8 \rrbracket$ & 0 & \\
\hline Diabetes mellitus with end-organ damage & $2 \varangle 0.8 \rrbracket$ & $1(2.2)$ & 0.353 \\
\hline Renal disease & $54 \llbracket 20.4 \rrbracket$ & 13(28.9) & 0.200 \\
\hline Total Charlson score & $2(0-10)$ & $3(0-8)$ & 0.186 \\
\hline Creatinine $(\mathrm{mg} / \mathrm{dL})^{\mathrm{a}}$ & $65.5(21-926.8)$ & 70.55(18.6-463.3) & 0.460 \\
\hline Albumin $(\mathrm{g} / \mathrm{dL})^{\mathrm{a}}$ & $33.9(3.9-62.8)$ & $32.65(16.5-45.9)$ & 0.129 \\
\hline WBC $(\times 109 / L)^{a}$ & $11.17 \pm 5.20$ & $11.07 \pm 4.60$ & 0.906 \\
\hline Systolic blood pressure $(\mathrm{mm} \mathrm{Hg})^{\mathrm{a}}$ & $124.09 \pm 18.39$ & $122.94 \pm 16.15$ & 0.520 \\
\hline Hemodynamic support ${ }^{\mathrm{a}}$ & $151 \otimes 57.0 \otimes$ & $21(46.7)$ & 0.208 \\
\hline Invasive ventilator support ${ }^{a}$ & $163 \rrbracket 61.5 \rrbracket$ & $25(55.6)$ & 0.450 \\
\hline Normal consciousness & 137ه51.7凶 & 13(28.9) & 0.045 \\
\hline
\end{tabular}




\begin{tabular}{|c|c|c|c|}
\hline Arterial line & $58 \rrbracket 21.9 \rrbracket$ & $14(31.1)$ & 0.302 \\
\hline Urine catheter & $202 \bigotimes 76.2 \rrbracket$ & $36(80.0)$ & 0.580 \\
\hline Central line & $122 \rrbracket 46.0 \rrbracket$ & $23(51.1)$ & 0.529 \\
\hline Nasogastric tube & 209ه78.9ه & $35(77.8)$ & 0.869 \\
\hline Acquisition in ICU & 140囚52.8ه & $25(55.6)$ & 0.735 \\
\hline Recent surgery & $119 \llbracket 44.9 \rrbracket$ & $13(28.9)$ & 0.045 \\
\hline Type of bacteria & & & 0.992 \\
\hline Acinetobacter & 196冈74.0ه & $33(73.3)$ & \\
\hline Enterobacteriaceae (Klebsiella) & $29 ه 10.9 \rrbracket$ & $6(13.3)$ & \\
\hline Pseudomonas/other & 40囚15.1凶 & $6(13.3)$ & \\
\hline EAT & & & 0.447 \\
\hline REAT & $67 \rrbracket 25.3 \rrbracket$ & $9(20.0)$ & \\
\hline UEAT & 198囚74.7囚 & $36(80.0)$ & \\
\hline REAT by time & & & 0.876 \\
\hline Same day as culture & $48 \rrbracket 18.1 \rrbracket$ & $7(15.6)$ & \\
\hline Day +1 & $9 \llbracket 3.4 \rrbracket$ & $1(2.2)$ & \\
\hline Day +2 & $7 \rrbracket 2.6 \rrbracket$ & $1(2.2)$ & \\
\hline
\end{tabular}

Data are presented as No. (\%) unless otherwise indicated.

aat culture taken day,

Abbreviations: BMI, body mass index; WBC, white blood cell; EAT: empirical antibiotic treatment; REAT: reasonable empirical antibiotic treatment; UEAT: unreasonable empirical antibiotic treatment; ICU, intensive care unit.

\section{Risk Factors for 30-day Mortality}

The total 30 day mortality rate was $16.98 \%(45 / 265)$, 9 out of 76 patients with REAT (11.84\%) died, compared with $36(15.38 \%)$ of 234 patients with UEAT $(P=0.447)$. In univariate analysis(table 6$)$. congestive heart failure, dementia, unconsciousness, and recent surgery were risk factors for 30-day mortality, while other factors had no significant effect on the survival of patients. In addition, we classified EAT according to the time, and there was no significant effect on the survival of patients who received EAT at different time $(P=0.876)$. Moreover, EAT and significant risk factors $(P<0.05$,Table 6$)$ of 30-day mortality were analyzed by multivariable regression analysis. As shown in Table 7, after 
controlling covariates, UEAT was not a risk factor for 30-day mortality (OR0.876, Cl95\% 0.377-2.040, P $0.760)$.

Table 7. Risk Factors for 30-day Mortality, Multivariate Analysis

\begin{tabular}{|lllll|}
\hline & \multicolumn{5}{c|}{$95 \% \mathrm{Cl}$} \\
\hline Factor & OR & Lower & Upper & $\mathrm{P}$ \\
\hline Congestive heart failure & 0.553 & 0.266 & 1.148 & 0.112 \\
\hline Dementia & 0.125 & 0.025 & 0.629 & 0.012 \\
\hline Normal consciousness & 2.538 & 1.236 & 5.213 & 0.011 \\
\hline Recent surgery & 1.991 & 0.972 & 4.077 & 0.06 \\
\hline REAT & 0.876 & 0.377 & 2.04 & 0.76 \\
\hline
\end{tabular}

Abbreviations: Cl: confdence interval; OR: odds ratio; REAT: reasonable empirical antibiotic treatment.

\section{Discussion}

In this work, we found that whether in univariate or multivariate analysis, the final results were consistent and stable, that was, UEAT did not increase the 30-day mortality rate, while it increased length of hospital stay. Above data showed that UEAT prolonged the length of stay for about 2.5 days, the antibiotic regimens used in this study almost were combined therapy. This result only concerned patients with CRGNB-infected hospital-acquired pneumonia, and the main bacteria were Acinetobacter baumannii $(68 \%)$. The same results had been found in previous studies ${ }^{[13-15]}$. However, in other studies, we found that there were disagreement with our conclusion that UEAT could increase 30 -day mortality rate ${ }^{[16-20]}$. This may be due to the high drug resistance rate of CRGNB which lead to serious illness and high mortality rate for CRGNB-infected pneumonia. This high mortality rate may real the role of REAT. But it does not mean that REAT has no effect on 30-day mortality. It may be that REAT can reduce 30-day mortality and hospitalization time of inpatients with mild and moderate diseases but has no obvious effect on severe patients ${ }^{[21]}$. Besides, in the study of Michek ST ${ }^{[22]}$, it was found that UEAT did not increase mortality in patients with early-onset infection, but increased mortality in patients with late-onset infection. Therefore, there was no difference between UEAT and REAT in 30-day mortality in this article. In addition, other possible reasons for this result may lie in the differences of research type, age distribution, region, bacterial distribution, research object, sample size, variable control and so on.

In the analysis of hospital stay, pulmonary disease, hemodynamic support at culture taken day and recent surgery are risk factors for hospital stay, which suggests that inpatients with pulmonary disease, hemodynamic support at culture taken day and recent surgery will stay longer. UEAT could extend hospitalization samely. Therefore, clinicians should pay more attention to REAT to reduce the length of stay of patients. Moveover, the results of catheter insertion analysis show that whether catheter, venous 
catheter, arterial catheter, ventilator or nasal catheter is not a risk factor for hospital stay. which is also different from other literature ${ }^{[23]}$. The possible reason for this result is that the patient's serious condition does not reflect the influence of mechanical ventilation.

For the analysis of mortality, dementia, unconsciousness are risk factors for 30-day mortality, Clinicians can assess patients' survival status based on this result and thus provide corresponding survival support. In the analysis of REAT by time, we did not find any difference in mortality rate at different time in the early stage. However, literature reports that the evaluation of 72-hour empirical therapy was significantly correlated with the improvement of treatment rate, duration of antibiotic treatment and shortening of hospitalization time $\mathrm{e}^{[23,24]}$. The possible reason is that the number of our cases is too small to reflect the real results.

The advantages of our experiment are as follows: firstly, we analyzed a number of variables that may have impact on 30-day mortality and hospital stay, including patient demographic statistics, basic diseases, test results, infection and so on. Secondly, we controlled other variables and analyzed the influence of duration of hospitalization on 30-day mortality by multivariable regression analysis. Finally, we classified experiential therapy according to time and analyzed the experimental data in many directions, which give direct and convenient results.

The experimental shortcomings including the experimental data are limited to the situation of hospitalacquired bacterial pneumonia in one hospital in recent years, there is no comprehensive evaluation of hospitals in different regions. Moreover, the experiment is limited to the hospital-acquired bacterial pneumonia by CRGNB. The situation of community-acquired pneumonia, bloodstream infection and other infections are not clear. In the selection of variables, only some of variables are selected. As we all know, in clinical death cases, any step and environment of patient's life can affect patient's survival, including economic status, medical environment, nursing situation, patient's psychological status and any other aspects. In the research of REAT according to time classification, our total data are less, which may not reflect the real situation, so further research is needed. And the results only assessed the impact of EAT on 30-day mortality and length of stay. Obviously, the causes of death of patients are not only infection and empirical therapy can explain. In addition, there are literature focusing on the classification of therapeutic drugs of empirical antibiotics, including the effects of antibiotics alone and combinedly, which are not reflected in our experiments. The average age of the cases in the experiment was about 60 years old, which was limited to the comparison of the cases in the higher age group. According to chart 2, Acinetobacter baumannii accounted for a large proportion of cases in the random test analysis, which only represented the results of this study. Furthermore, emergency department patients accounted for $55.2 \%(171 / 310)$ in this study.

In conclusion, for severe pneumonia with hospital-acquired CRGNB infection, UEAT does not increase the 30-day mortality rate, while increase the length of hospitalization. At the same time, the excessive or unreasonable use of antibiotics were related to the increase of bacterial resistance, side effects and treatment costs. Considering this, we recommend clinicians give REAT in the treatment of infection. 


\section{Declarations}

Funding. This study was funded by Research Fund of Anhui Institute of Translational Medicine(Immunoprotection of His-A12(1-85) recombinant protein of Pneumocystis carinii) (grant number 2017zhyx14).

Conflict of Interest. The authors declare that they have no conflict of interest.

Ethical approval. All procedures performed in studies involving human participants were in accordance with the ethical standards of the medical ethics committee of the First Affiliated Hospital of Anhui Medical University (the reference number:Quick-PJ 2019-12-18) and with the 1964 Helsinki declaration and its later amendments or comparable ethical standards

Informed consent. Informed consent was obtained from all individual participants included in the study.

Disclosure. None of the authors have any personal or financial involvement with the organizations that have financial interest in its content. The authors report no conflicts of interest in this work.

Author contributions. All authors contributed to the study conception and design. Material preparation, data collection and analysis were performed by Rongrong Li, Jilu Shen. The first draft of the manuscript was written by Rongrong Li and all authors commented on previous versions of the manuscript. All authors read and approved the final manuscriptReferences

\section{References}

1. Paul M, Andreassen S, Tacconelli E, et al. Improving empirical antibiotic treatment using TREAT, a computerized decision support system:cluster randomized trial. Antimicrob Chemother, 2006; 58(6):1238-45.

2. SligI WI, Dragan T, Smith SW. Nosocomial Gram-negative bacteremia in intensive care: epidemiology, antimicrobial susceptibilities, and outcomes. Int J Infect Dis,2015; 37(8):129-34.

3. Melzer M, Welch C. 30-day mortality in UK patients with bacteraemic community-acquired pneumonia. Infection, 2013; 41(5):1005-11.

4. Carrara E, Pfeffer I, Zusman O, et al. Determinants of inappropriate empirical antibiotic treatment: systematic review and meta-analysis. Int J Antimicrob Agents, 2018; 51(4):548-53.

5. Park SY, Park HJ, Moon SM, et al. Impact of adequate empirical combination therapy on mortalit from bacteremic Pseudomonas aeruginosa pneumonia. BMC infectious diseases, 2012; 12(11):308.

6. Zak-Doron Y, Dishon Benattar Y, Pfeffer I, et al. The Association Between Empirical Antibiotic Treatment and Mortality in Severe Infections Caused by Carbapenem-resistant Gram-negative Bacteria:A Prospective Study. Clinical Infectious Diseases, 2018; 67(12):1815-1823.

7. Kang $\mathrm{Cl}$, Wi YM, Ko KS, et al. Outcomes and risk factors for mortality in community-onset bacteremia caused by extended-spectrum beta-lactamase-producing Escherichia coli, with a special emphasis 
on antimicrobial therapy. Scand J Infect Dis, 2013; 45(7):519-25.

8. Eliakim-Raz N, Babitch T, Shaw E, et al. Risk Factors for Treatment Failure and Mortality Among Hospitalized Patients With Complicated Urinary Tract Infection: A Multicenter Retrospective Cohort Study (RESCUING Study Group). Clin Infect Dis,2019; 68(1):29-36.

9. Yoon YK, Park DW, Sohn JW, et al. Effects of inappropriate empirical antibiotic therapy on mortality in patients with healthcare-associated methicillin-resistant Staphylococcus aureus bacteremia: a propensity-matched analysis. BMC Infect Dis,2016; 16(7):331.

10. Kalil AC, Metersky ML, Klompas M, et al. Executive Summary: Management of Adults With Hospitalacquired and Ventilator-associated Pneumonia: 2016 Clinical Practice Guidelines by the Infectious Diseases Society of America and the American Thoracic Society. Clinical Infectious Diseases, 2016; 63(5):575-82.

11. Longfei Guo, Fengjing Hu, Ling Cai, et al. Current status and risk assessment of nosocomial infections in critical medicine. Chinese Journal Of Nosocomology, 2019; 29(08):1263-1266.

12. Xu Y, Lai C, Xu G, et al. Risk factors of ventilator-associated pneumonia in elderly patients receiving mechanical ventilation. Clin Interv Aging,2019; 14(6):1027-103.

13. Gutiérrez-Gutiérrez $B$, Salamanca $E$, de Cueto $M$, et al. A predictive model of mortality in patients with bloodstream infections due to carbapenemase-producing Enterobacteriaceae. Mayo Clinic Proceedings, 2016; 91(10):1362-1371.

14. Lim CL, Spelman D. Mortality impact of empirical antimicrobial therapy in ESBL-and AmpCproducing Enterobacteriaceae bacteremia in an Australian tertiary hospital. Infect Dis Health,2019; 24(3): 124-133.

15. Wiggers JB, Sehgal P, Pinto R, et al. The association of adequate empirical treatment and time to recovery from bacteraemic urinary tract infections: a retrospective cohort study. Clin Microbiol Infect, 2019; 25:1253-1258.

16. Kim YJ, Jun YH, Kim YR, et al. Risk factors for mortality in patients with Pseudomonas aeruginosa bacteremia; retrospective study of impact of combination antimicrobial therapy. BMC infectious diseases, 2014; 14(3):161.

17. Cheng WL, Hsueh PR, Lee CC, et al. Bacteremic pneumonia caused by extended-spectrum betalactamase-producing Escherichia coli and Klebsiella pneumoniae: Appropriateness of empirical treatment matters, J Microbiol Immunol Infect.2016; 49(2):208-15 .

18. Al-Dorzi HM, Asiri AM, Shimemri A, et al. Impact of empirical antimicrobial therapy on the outcome of critically ill patients with Acinetobacter bacteremia. Annals Of Thoracic Medicine, 2015; 10(4):25662.

19. Park SY, Park HJ, Moon SM, et al. Impact of adequate empirical combination therapy on mortality from bacteremic Pseudomonas aeruginosa pneumonia. BMC Infectious Diseases,2012; 12(11):308.

20. Neuner EA, Yeh JY, Hall GS, et al. Treatment and outcomes in carbapenem-resistant Klebsiella pneumoniae bloodstream infections. Diagnostic Microbiology And Infecticous Disease,2011; 69(4):357-62. 
21. null null, Bertolini G, Nattino G, et al. Mortality attributable to different Klebsiella susceptibility patterns and to the coverage of empirical antibiotic therapy: a cohort study on patients admitted to the ICU with infection. Intensive Care Med,2018; 44(10):1709-1719.

22. Micek ST, Hampton N. Risk Factors and Outcomes for Ineffective Empiric Treatment of Sepsis Caused by Gram-Negative Pathogens: Stratification by Onset of Infection[J]. Antimicrobial agents and chemotherapy, 2018; 62(1):e01577-17.

23. Zak-Doron Y, Dishon Benattar Y, Pfeffer I, et al. The Association Between Empirical Antibiotic Treatment and Mortality in Severe Infections Caused by Carbapenem-resistant Gram-negative Bacteria:A Prospective Study. Clinical Infectious Diseases.2018; 67(12):1815-1823.

24. Murri R, Taccari F, Spanu T, et al. A 72-h intervention for improvement of the rate of optimal antibiotic therapy in patients with bloodstream infections. Eur J Clin Microbiol Infect Dis, 2018; 37:167-173. 